\title{
BMJ Open Policy changes and the screening, diagnosis and treatment of drug- resistant tuberculosis patients from 2015 to 2018 in Zhejiang Province, China: a retrospective cohort study
}

\author{
Weixi Jiang (D , ${ }^{1}$ Ying Peng, ${ }^{2}$ Xiaomeng Wang, ${ }^{2}$ Chris Elbers, ${ }^{3}$ Shenglan Tang, ${ }^{4}$ \\ Fei Huang, ${ }^{5}$ Bin Chen, ${ }^{2}$ Frank Cobelens ${ }^{6}$
}

To cite: Jiang W, Peng $Y$, Wang $X$, et al. Policy changes and the screening, diagnosis and treatment of drug-resistant tuberculosis patients from 2015 to 2018 in Zhejiang Province, China: a retrospective cohort study. BMJ Open 2021;11:e047023. doi:10.1136/ bmjopen-2020-047023

- Prepublication history and additional material for this paper is available online. To view these files, please visit the journal online (http://dx.doi.org/10. 1136/bmjopen-2020-047023)

Received 18 November 2020 Revised 02 March 2021 Accepted 24 March 2021
Check for updates

(C) Author(s) (or their employer(s)) 2021. Re-use permitted under CC BY. Published by BMJ.

For numbered affiliations see end of article.

Correspondence to Mr Bin Chen; bchen@cdc.zj.cn

\section{ABSTRACT}

Objectives To examine changes in the screening, diagnosis, treatment and management of drug-resistant tuberculosis (DRTB) patients, and investigate the impacts of DRTB-related policies on patients of different demographic and socioeconomic characteristics.

Design A retrospective cohort study using registry data, plus a survey on DRTB-related policies.

Setting All prefecture-level Centres for Disease Control in Zhejiang Province, China.

Main outcome measures Alongside the care cascade, we examined: (1) reported number of presumptive DRTB patients; (2) percentage of presumptive patients with drug susceptibility testing (DST) records; (3) percentage of DRTB/rifampicin-resistant (RR) patients registered; (4) percentage of RR/multidrug-resistant TB (MDRTB) patients that received anti-DRTB treatment; and (5) percentage of RR/MDRTB patients cured/completed treatment among those treated. Multivariate logistic regressions were conducted to explore the impacts of DRTB policies after adjusting for other factors.

Results The number of reported presumptive DRTB patients and the percentage with DST records largely increased during 2015-2018, and the percentage of registered patients who received anti-DRTB treatment also increased from $59.0 \%$ to $86.5 \%$. Patients under the policies of equipping GeneXpert plus expanded criteria for DST had a higher likelihood of being registered compared with no GeneXpert (adjusted OR (aOR) $=2.57,95 \% \mathrm{Cl}: 1.20$ to 5.51), while for treatment initiation the association was only significant when further expanding the registration criteria $(\mathrm{aOR}=2.38,95 \% \mathrm{Cl}: 1.19$ to 4.79). Patients with registered residence inside Zhejiang were more likely to be registered (aOR=1.96, 95\% Cl: 1.52 to 2.52), treated (aOR=3.83, $95 \% \mathrm{Cl}: 2.78$ to 5.28$)$ and complete treatment $(\mathrm{aOR}=1.92$, $95 \% \mathrm{Cl}: 1.03$ to 3.59 ) compared with those outside.

Conclusion The policy changes on DST and registration have effectively improved DRTB case finding and care. Nevertheless, challenges remain in servicing vulnerable groups such as migrants and improving equity in the access to TB care. Future policies should provide comprehensive support for migrants to complete treatment at their current place of residence.

\section{Strengths and limitations of this study}

- This study conducted a comprehensive and systematic cascade analysis on the healthcare pathway for drug-resistant tuberculosis (DRTB) patients: screening, diagnosis, treatment and management, and examined key factors associated with the case finding and healthcare process.

- This study evaluated the effectiveness of different combinations of DRTB-related policies as they were implemented in all prefectures of one Chinese province, including new diagnostic technology promotion, improved patient management and financial support.

- This study also examined those patients left-behind during the policy changes from an equity perspective, with a focus on rifampin monoresistant patients and patients with registered residence (Hukou) outside Zhejiang.

- The impacts of the financing policies could not be explored as almost all prefectures had some form of financing policies since 2015 and there were no individual-level data on whether the patient benefited from these financing policies.

- Other potentially important socioeconomic factors like education and income were not available in the dataset used, limiting, therefore, the scope of the equity analysis to migrant workers.

\section{INTRODUCTION}

Drug-resistant tuberculosis (DRTB) has become a global concern in recent years. It is estimated that globally in $2018,3.4 \%$ of new $\mathrm{TB}$ patients and $18 \%$ of previously treated patients developed rifampicin-resistant (RR) forms of disease, including multidrugresistant TB (MDRTB), in which there is additional resistance to isoniazid, and $7.1 \%$ and $21 \%$, respectively, in China. ${ }^{1}$ Currently, China accounts for $14 \%$ of the RR/MDRTB disease burden, and one recent projection suggested 
the incidence of RR/MDRTB would triple without interventions to change current conditions. ${ }^{2}$ The RR/MDRTB epidemic has posed a great challenge to achieve the target of Sustainable Development Goals (SDGs) of ending TB in 2030 in China. ${ }^{3}$

The diagnosis and treatment of DRTB can be very costly in terms of both time and money, ${ }^{4}$ and barriers to accessing DRTB diagnosis and care exist worldwide. ${ }^{5}$ The diagnosis of DRTB takes 1-3 months using traditional technology, and the treatment of RR/MDRTB lasts for up to 2 years. DRTB patients generally have lower socioeconomic status, and the cost of treatment is so high that the current financing policies in the form of health insurance reimbursement and subsidised treatment are far from sufficient. ${ }^{6-8}$ For China specifically, the public health insurance programmes provide very limited coverage for outpatient services that are required for around 20 months for RR/MDRTB patients. In addition, some second-line anti-TB drugs and auxiliary drugs are often not covered. ${ }^{9-11}$ Previous studies in several countries, including China, have also revealed long treatment delay, high pre-diagnosis and pre-treatment attrition, and high loss-to-follow-up during the treatment course. ${ }^{12-17}$ Moreover, the MDRTB treatment success rate in China was less than $50 \% .^{18}$

These formidable barriers for DRTB patients to accessing and adhering to standard treatment call for strong supporting policies for patients to receive and complete treatment. ${ }^{19}$ Previous studies have validated the utility of rapid drug susceptibility testing (DST) technologies such as Genechip and GeneXpert in the screening for DRTB, and shown that these technologies could improve DRTB case finding, shorten treatment delays and decrease pre-diagnosis attrition. ${ }^{1320-24}$ As for treatment adherence, one comprehensive programme in China that provided universal health coverage to MDRTB patients was shown to improve access to and affordability of diagnosis and quality treatment of MDRTB. ${ }^{5}$ Studies on other intervention strategies showed that directly observed therapy or other reminding approaches through digital technologies could improve the treatment adherence and outcomes of TB/MDRTB. ${ }^{25}{ }^{26}$ Patient counselling alone or combined with financial support can also increase the cure rates among MDRTB patients. ${ }^{27}$

In China, some provinces, along with or after the roll-out of international donor-funded projects, have implemented policies to improve DRTB control, including allocating special funds to equip DRTB designated hospitals with DST facilities and reagents, improving health insurance benefit packages and providing subsidies to patients. ${ }^{1128}$ However, there is limited evidence on the impact of implementing these policies on DRTB case finding and care thereafter. The equity issues underlying the case finding and care procedures for DRTB patients are also understudied, especially considering that the eligibility for policies issued in a certain region is often linked with patients' registered residence, work and health insurance status.
This study aims to examine changes in the programmatic performance with regard to screening, diagnosis, treatment and management of DRTB patients in Zhejiang from 2015 to 2018 through a cascade analysis approach. ${ }^{29}$ In addition, as the policies and guidelines on this whole procedure of DRTB care changed during this period, this study systematically summarises these changes and investigates how these policy changes have influenced case finding and treatment of DRTB patients. We also explore whether these policy changes have equally influenced patients of different demographic and socioeconomic characteristics.

\section{METHODS \\ Study settings}

Zhejiang is a province located in the eastern area with its Gross Domestic Product (GDP) ranking fourth in China, ${ }^{30}$ and has a growing migrant population in recent years. Under the current TB control model in Zhejiang, the prefecture-level designated hospital, usually one in each prefecture, is responsible for the diagnosis and treatment of DRTB patients, while the Centers for Disease Control and Prevention (CDC) and primary healthcare facilities conduct patient management. Patients who are clinically suspected to have DRTB are to be referred for DST and should be reported in the Tuberculosis Information Management System (TBIMS) as presumptive DRTB patients. Their sputum samples are to be sent to the prefecture-level designated hospital for DST. If diagnosed with DRTB, these patients should be registered in the TBIMS database for diagnosed patients as such. The criteria regarding what types of DRTB should be registered have being changing over time.

\section{Study design}

This study included a questionnaire survey on the DRTB policies/programmes among the 11 prefecture-level CDCs and a quantitative analysis of the TBIMS records of presumptive and diagnosed DRTB patients.

\section{Questionnaire survey on DRTB policies/programmes}

A questionnaire on the DRTB-related policies was distributed to the CDC of all prefectures in Zhejiang in collaboration with Zhejiang provincial CDC. After preliminary consultation with the provincial CDC, the questionnaire was designed to include policies in four areas: (1) eligibility of presumptive DRTB patients for DST; (2) eligibility for registering DRTB patients based on the type of drug resistance (including rifampin monoresistance (RMR), MDR, extensive drug resistance (XDR) and monoresistance to other types of drugs); (3) DST technology and payment for DST and (4) financing policies, including both health insurance benefit packages and government subsidies for supporting the DRTB treatment. Detailed questions on the eligibility for financial support regarding the types of DRTB, the registered permanent residence and the region of health insurance enrolment were also included 
in the questionnaire (see online supplemental material 1 ). If the policies had changed at any time after 2015, we collected information on the policy details before and after the change as well as the year of change.

\section{TBIMS records}

De-identified TBIMS records of presumptive and diagnosed DRTB patients from 2015 to 2018 in Zhejiang were retrieved from the National Center for Tuberculosis Control and Prevention of China, CDC. The dataset of presumptive DRTB patients included demographic information (prefecture of registration, age, sex, ethnicity, occupation and registered residence), drug-resistance test profile (the date of sending the sample, conducting the test and reporting the result, the type of test and the test result), as well as a unique registration number if registered as a DRTB patient in the diagnosed patient dataset. Only those DRTB patients recorded in the diagnosed patient dataset have a traceable treatment history and are managed under a specialised guideline for DRTB patients. The dataset of diagnosed DRTB patients contained the same demographic information and diagnostic data as the dataset of presumptive patients, plus the treatment information, including the starting date of anti-DRTB treatment, including both second-line $(\mathrm{RR} / \mathrm{MDRTB})$ and adapted first-line (monoresistance to isoniazid) treatment, TB treatment history, current state of treatment (under treatment or ended) and the ending date of treatment as well as the reason of ending the treatment (cured/treatment completed/death/lostto-follow-up/others) if ended. In TBIMS, the treatment status referred to whether the patient received treatment in Zhejiang. If patients were registered but returned to their hometown for treatment, it could be shown as 'treatment refused'.

\section{Patient and public involvement statement}

Patients or the public were not involved in the design, or conduct, or reporting, or dissemination plans of our research.

\section{Data analysis}

Using datasets of the presumptive and registered diagnosed patients, a seven-step cascade of diagnosis and care was constructed for each year from 2015 to 2018 : (1) reported presumptive DRTB patients; (2) presumptive patients who had DST; (3) diagnosed DRTB, and RR/ MDRTB (including RMR, MDR and XDR) patients; (4) registered RR/MDRTB patients; (5) RR/MDRTB patients that ever received anti-DRTB treatment; (6) RR/MDRTB patients that were under treatment 6 months after treatment initiated; and (7) RR/MDRTB patients that had been cured or completed treatment, as shown in figure 1 . Diagnosed DRTB patients were defined as patients with a DST result showing resistance to at least one type of first-line anti-TB drug. As in 10 out of the 11 prefectures only RR/MDRTB patients were required to be registered and thus had available data on treatment history before 2019, we only analysed RR/MDRTB patients for steps 4-7. Descriptive analyses were conducted to explore the reasons for attrition at each step based on the relevant records in the datasets. Patients' ethnicity was categorised as Han and other minority groups. Frequencies of patient characteristics were also calculated across patient groups at each step of the cascade to examine potential factors associated with the attrition. For steps $3-5$, the frequencies of some patient characteristics of interests, including age, sex, drug-resistant type, occupation and registered residence, were calculated separately for each year to examine the trends from 2015 to 2018.

Mixed-effect two-level logistic regressions, with fixed slope and random intercept specification, were conducted to explore factors associated with the likelihood of being registered for RRTB patients, the likelihood of receiving anti-DRTB treatment if registered and the likelihood of getting cured or completing treatment (the latter only for those who initiated treatment before 2017 because of the 2-year treatment duration). This model showed better fit to the data than the fixed-effect model based on the likelihood-ratio test. The DRTB-related policies were summarised along the four areas in the questionnaires. Besides patient-level variables, the policies implemented in each prefecture and each year, categorised according to the different combinations of policies in the four areas, were included in the model. Per-capita GDP of the prefecture was divided into three groups, as the numbers assembled in three intervals: RMB 55000-70

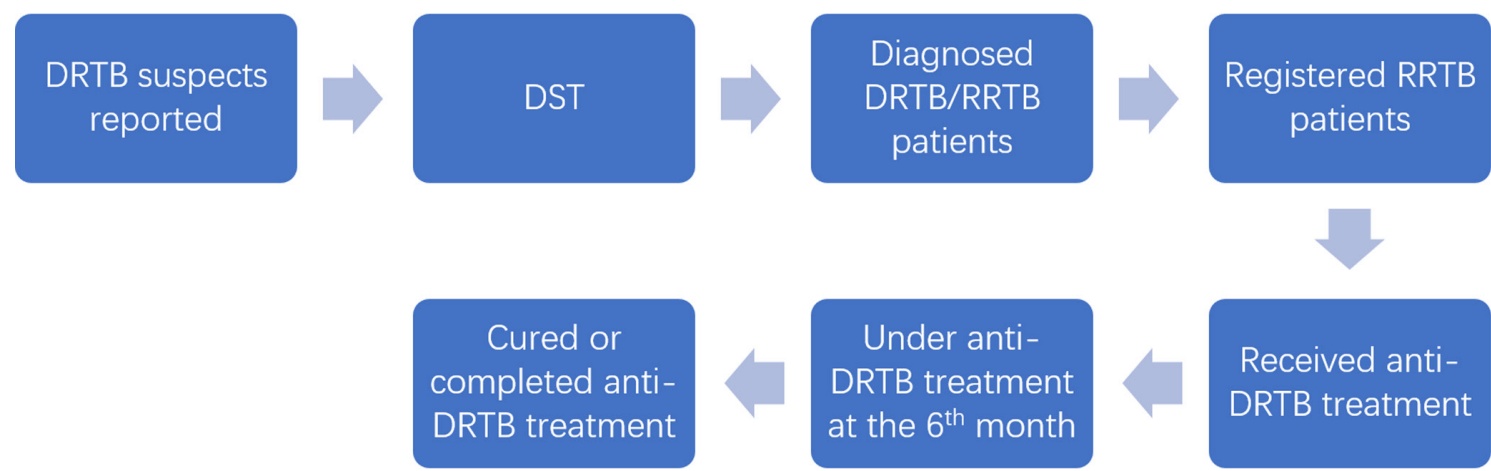

Figure 1 Seven steps of the DRTB diagnosis and care cascade. DRTB, drug-resistant tuberculosis; RRTB, rifampicin-resistant tuberculosis. 
000, RMB $80000-100000$ and above RMB 120 000, and also included in the model as a categorical variable. Standard errors were estimated accounting for clustering, considering the intra-prefecture correlations.

\section{RESULTS}

\section{DRTB policy change from 2015 to 2018 in Zhejiang}

Results from the questionnaire survey on DRTB policies showed that the major change in the eligibility of patients for DST was the expansion from high-risk patients (including smear positive relapse, treatment failure and positive sputum bacteriology after 2 months' treatment), to all bacteriologically positive TB patients. As for the testing technology, the major change was the introduction of the rapid DST assay GeneXpert MTB/ RIF that combines testing for Mycobacterium tuberculosis with screening for rifampicin-resistance with a same-day result, while the costs for the test were all covered, or mostly covered by the government. The criteria for DRTB patient registration (implying eligibility for specialised DRTB patient management) also expanded from MDR/ XDRTB to RRTB, and one prefecture even expanded to any DRTB before 2018. In most prefectures, these changes in DST eligibility and equipment as well as the registration criteria happened in 2017, while almost all others had adopted the new polices and introduced GeneXpert earlier (online supplemental table 1).

The financing policies to support DRTB treatment in Zhejiang consisted of both health insurance and medical assistance policies, and in most prefectures, these policies had been implemented in 2015 . These financing policies aimed to improve the benefit package for DRTB patients through a three-layer coverage system: (1) increasing the reimbursement rate of outpatient treatment to $70 \%-90 \%$, the same level as for inpatient treatment; (2) the out-ofpocket (OOP) expenditure exceeding a certain amount, typically RMB 20000-30 000, could be further reimbursed at a rate of $55 \%-85 \%$ through a supplemental health insurance programme for critical illnesses; (3) the OOP expenditure after health insurance reimbursement could be covered by the medical assistance, with a payment limit varying from RMB 11000 to over RMB 60 000 across prefectures. As for the eligibility for the insurance policies, those with public health insurance enrolled outside Zhejiang, usually migrants without formal jobs, were excluded. In 5 out of the 11 prefectures, RMR patients were still not eligible for the expanded public health insurance benefit package up to 2019. For the medical assistance, in 6 out of the 11 prefectures, only patients with registered residence ('Hukou') in Zhejiang were eligible, and for the other 4 prefectures with assistance policies, the eligibility had been expanded to all residents in this prefecture. Up to 2018, RMR patients in five prefectures were still excluded from the medical assistance (online supplemental table 2).

\section{Cascade analysis of DRTB care from 2015 to 2018}

Table 1 shows the screening and diagnosis of DRTB patients from 2015 to 2018. In general, the results suggested increased capacity to find DRTB patients. The number of reported presumptive DRTB patients increased from 9285 to 23 916, with the largest increase from 2016 to 2017 coinciding with the change in screening policy in most prefectures. The percentage of patients with test results also increased from $69.3 \%$ to $78.1 \%$. Along with the increase in the volume of DST, there was an increase in the number of diagnosed DRTB patients except for 2018, and a decrease in the percentage of patients diagnosed among those tested. RR/MDRTB patients accounted for around $40 \%-50 \%$ of the total DRTB patients.

Further analysis on the types of diagnostic tests patients received revealed an increasing trend in the percentage of patients taking rapid DST as well as the percentage of patients tested with both conventional culture-based and rapid DST. The percentage of presumptive patients without DST profile for unknown reasons as recorded in the TBIMS dataset, which indicates pre-diagnosis attrition, dropped dramatically from $17.3 \%$ to $2.7 \%$ (online supplemental table 3 ).

Table 2 shows the registration and treatment for diagnosed RRTB patients from 2015 to 2018. While the actual number of registered RRTB patients increased, the percentage of registered RRTB patients dropped in 2017 , and this percentage increased again to $84.6 \%$. The percentage of registered patients that received antiDRTB treatment increased from $59.0 \%$ to $86.5 \%$, and the percentage of those treated who received at least 6-month treatment remained above $90 \%$. Around $70 \%$ of patients diagnosed in 2015 and 2016 completed treatment.

Table 1 Diagnostic cascade starting from presumptive DRTB patients

\begin{tabular}{|c|c|c|c|c|c|c|c|}
\hline \multirow[b]{2}{*}{ Year } & \multirow{2}{*}{$\begin{array}{l}\text { Number of presumptive DRTB } \\
\text { patients }\end{array}$} & \multicolumn{2}{|c|}{$\begin{array}{l}\text { Patients with test } \\
\text { records }\end{array}$} & \multicolumn{2}{|c|}{$\begin{array}{l}\text { Diagnosed DRTB } \\
\text { patients }\end{array}$} & \multicolumn{2}{|c|}{$\begin{array}{l}\text { Diagnosed RR/MDRTB } \\
\text { patients }\end{array}$} \\
\hline & & Number & $\%$ & Number & $\%$ & Number & $\%$ \\
\hline 2016 & 10997 & 8438 & 76.7 & 1258 & 14.9 & 529 & 42.1 \\
\hline 2017 & 21768 & 14764 & 67.8 & 1729 & 11.7 & 716 & 41.4 \\
\hline
\end{tabular}

DRTB, drug-resistant tuberculosis; MDRTB, multidrug-resistant tuberculosis; RR, rifampicin resistant. 
Table 2 Registration and treatment cascade for diagnosed RRTB patients

\begin{tabular}{|c|c|c|c|c|c|c|c|c|c|}
\hline \multirow[b]{2}{*}{ Year } & \multirow{2}{*}{$\begin{array}{l}\text { Diagnosed RR/ } \\
\text { MDRTB patients }\end{array}$} & \multicolumn{2}{|c|}{ Registered } & \multicolumn{2}{|c|}{$\begin{array}{l}\text { Received anti-DRTB } \\
\text { treatment }\end{array}$} & \multicolumn{2}{|c|}{$\begin{array}{l}\text { Under treatment } \\
6 \text { months after } \\
\text { treatment initiated }\end{array}$} & \multicolumn{2}{|c|}{$\begin{array}{l}\text { Cured or } \\
\text { completed } \\
\text { treatment }\end{array}$} \\
\hline & & No. & $\%$ & No. & $\%$ & No. & $\%$ & No. & $\%$ \\
\hline 2015 & 503 & 383 & 76.1 & 226 & 59.0 & 215 & 95.1 & 158 & 73.5 \\
\hline 2016 & 529 & 410 & 77.5 & 283 & 69.0 & 263 & 92.9 & 182 & 69.2 \\
\hline 2017 & 716 & 502 & 70.1 & 360 & 71.7 & 334 & 92.8 & - & - \\
\hline 2018 & 663 & 561 & 84.6 & 485 & 86.5 & 462 & 95.3 & - & - \\
\hline
\end{tabular}

DRTB, drug-resistant tuberculosis; MDRTB, multidrug-resistant tuberculosis; RRTB, rifampicin-resistant tuberculosis.

Duration of treatment could be longer than 2 years, as 38 out of the 283 patients starting treatment in 2016 were still shown as under treatment at the time we retrieved the data (see online supplemental table 4).

Factors associated with the registration and treatment of DRTB patients

Table 3 shows the characteristics of patients across each step of the care cascade. Around $70 \%$ of the presumptive and diagnosed patients were male, and around $98 \%$ of them were Han People. Only around $40 \%$ of the patients had formal jobs other than farming or unemployment. While under the assumption of perfect equity we would expect that the percentage of patients with different characteristics remained the same from the diagnosed to the treated group, it decreased from $30.3 \%$ to $27.0 \%$ for older patients, $28.1 \%$ to $22.5 \%$ for RMR patients, and

Table 3 Patient characteristics across each step of the care cascade

\begin{tabular}{|c|c|c|c|c|c|}
\hline & $\begin{array}{l}\text { Presumptive } \\
\text { DRTB patients }\end{array}$ & $\begin{array}{l}\text { Diagnosed } \\
\text { DRTB patients }\end{array}$ & $\begin{array}{l}\text { Diagnosed RR/ } \\
\text { MDRTB patients }\end{array}$ & $\begin{array}{l}\text { Registered RR/ } \\
\text { MDRTB patients }\end{array}$ & $\begin{array}{l}\text { RR/MDRTB } \\
\text { patients received } \\
\text { treatment }\end{array}$ \\
\hline $\mathrm{N}$ & 65966 & 5598 & 2411 & 1859 & 1357 \\
\hline \multicolumn{6}{|l|}{ Age (\%) } \\
\hline$\geq 60$ & 41.3 & 35.9 & 30.3 & 29.8 & 27.0 \\
\hline \multicolumn{6}{|l|}{ Gender (\%) } \\
\hline Male & 70.6 & 73.6 & 72.5 & 72.7 & 72.7 \\
\hline \multicolumn{6}{|l|}{ Ethnicity } \\
\hline Han (\%) & 97.7 & 98.0 & 97.9 & 98.0 & 98.8 \\
\hline \multicolumn{6}{|l|}{ Patient type (\%) } \\
\hline New patient & 70.2 & 65.2 & 51.8 & 51.4 & 48.8 \\
\hline \multicolumn{6}{|l|}{ Drug-resistant type (\%) } \\
\hline RMR & - & 12.1 & 28.1 & 20.8 & 22.5 \\
\hline MDR & - & 30.4 & 70.6 & 78.0 & 76.1 \\
\hline XDR & - & 0.5 & 1.2 & 1.2 & 1.4 \\
\hline Non-rifampicin resistant & - & 56.9 & - & - & - \\
\hline \multicolumn{6}{|l|}{ Registered residence (\%) } \\
\hline Outside Zhejiang & 23.4 & 25.2 & 24.4 & 22.3 & 17.0 \\
\hline \multicolumn{6}{|l|}{ Job category (\%) } \\
\hline Farmers & 49.0 & 48.0 & 43.8 & 45.0 & 45.5 \\
\hline Unemployed & 12.4 & 13.0 & 14.1 & 14.2 & 14.0 \\
\hline Other & 38.6 & 39.0 & 42.1 & 40.8 & 40.5 \\
\hline \multicolumn{6}{|l|}{ Test type (\%) } \\
\hline Fast test & - & 38.6 & 46.5 & 46.1 & 49.2 \\
\hline
\end{tabular}

DRTB, drug-resistant tuberculosis; MDRTB, multidrug-resistant tuberculosis; RMR, rifampin monoresistant; RR, rifamipicin resistant; XDR, extensive drug resistance. 

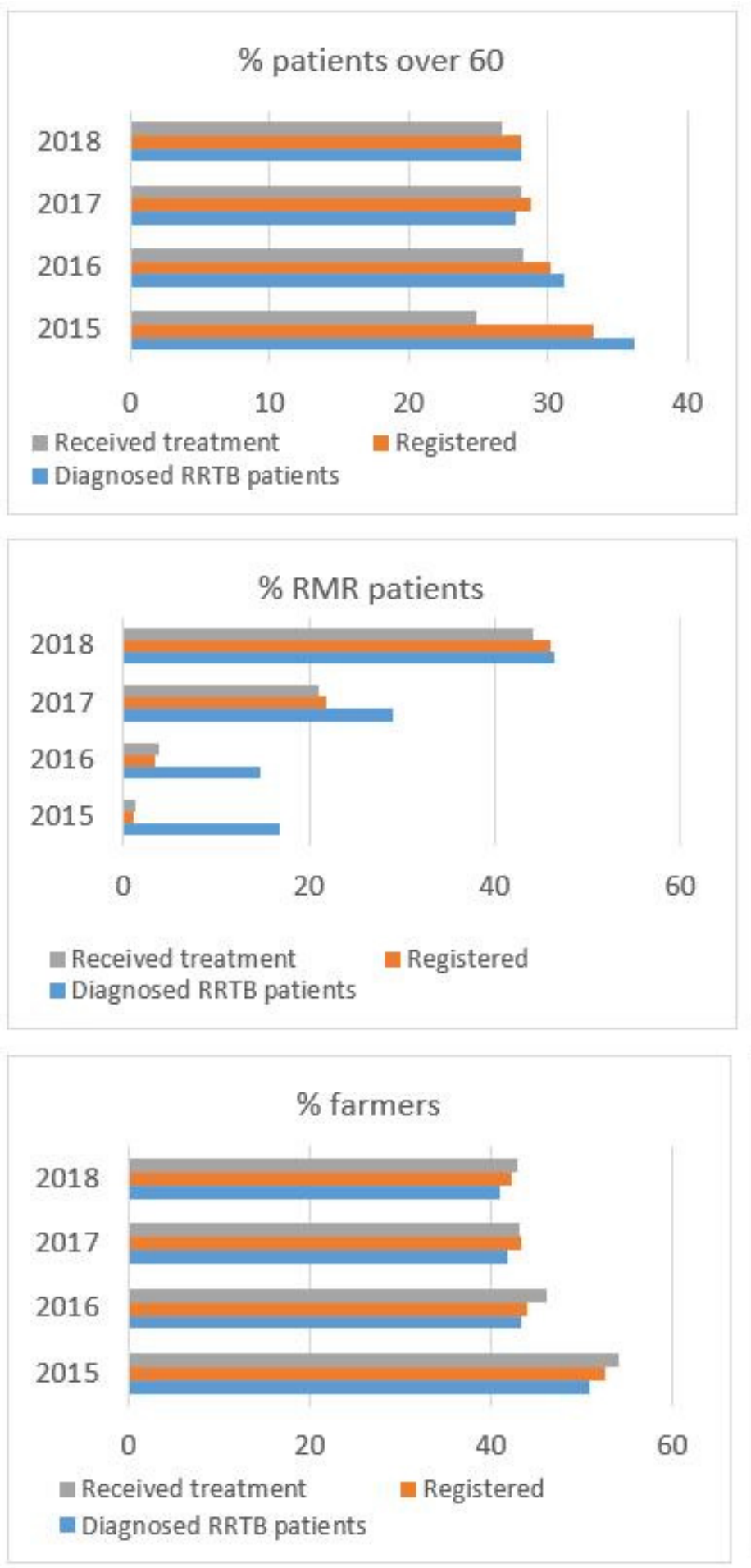
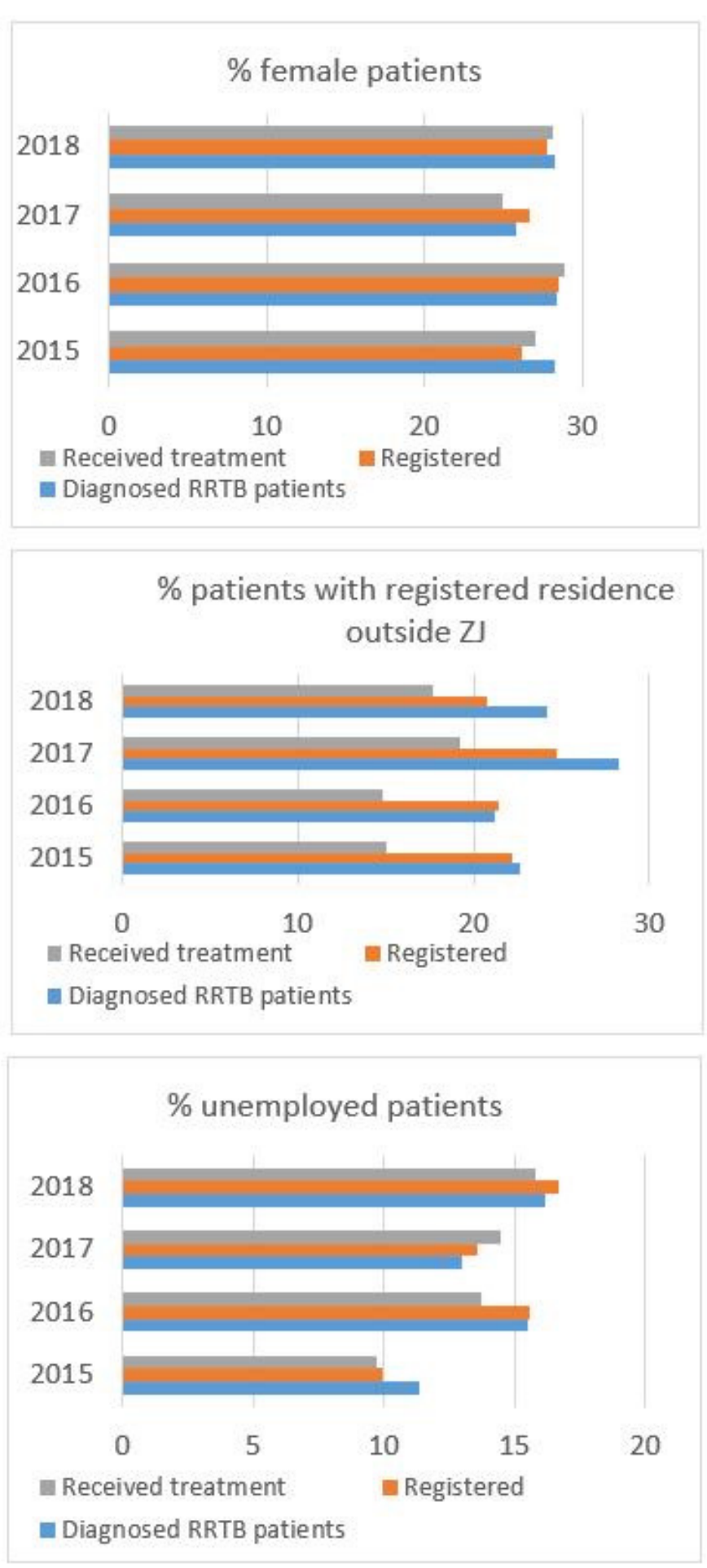

Figure 2 Characteristics of diagnosed, registered and treated RR/MDRTB patients, 2015-2018. RMR, rifampin monoresistant; RRTB, rifamipicin-resistant tuberculosis; ZJ, Zhejiang.

from $24.4 \%$ to $17.0 \%$ for patients with registered residence outside Zhejiang, that is, migrants. An increase was observed regarding the percentage of patients ever taking rapid DST.

Figure 2 shows the changes in the characteristics of diagnosed, registered and treated RR/MDRTB patients from 2015 to 2018. The percentage of diagnosed RMR patients that received adequate treatment was low in 2015 and
2016, but increased dramatically from 2017, coinciding with the policy change. The gaps between the proportion of older patients in the diagnosed and the treated group also narrowed. Nevertheless, over the 4years, there remained a $7 \%-10 \%$ decrease in the proportion of patients with registered residence outside Zhejiang from the diagnosed to the treated patient group, indicating that migrants were still more likely to drop out after 


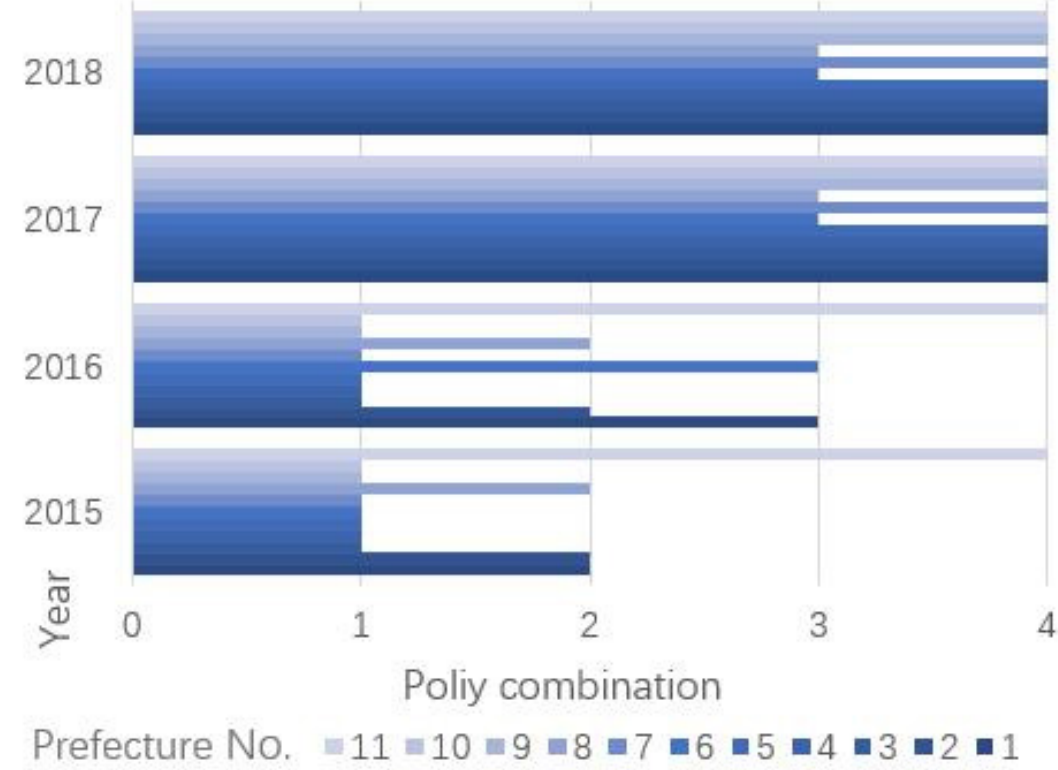

Policy combinations:

1: no GeneXpert

2: with GeneXpert, but no expansion of the criteria for DST

3: with GeneXpert + expanded critieria for DST, but no expansion of the registration eligibility to include RMR patients 4: 3 + expanding the registration eligibility to include RMR patients

Figure 3 Combination of DRTB policies implemented in each prefecture from 2015 to 2018. DST, drug susceptibility testing; RMR, rifampin monoresistant.

diagnosis compared with the local residents. Gender and job category were not obviously associated with the pretreatment attrition in this univariate analysis.

To systematically investigate the impact of policies on DRTB case finding and care, we divided the combination of policies in different areas of DRTB implemented in each prefecture for each year during 2015-2018 into four categories, considering both policy implications and the number of cases in each category. As shown in figure 3, since 2017, all prefectures had equipped GeneXpert and expanded the eligibility criteria for DST as well as registration, except for two prefectures which still did not register RMR patients.

Table 4 shows the results of mixed-effect two-level logistic regression of the factors associated with registration among diagnosed RRTB patients, as well as with receiving anti-DRTB treatment among those registered. After adjusting for other factors, patients under the policy of providing GeneXpert together with expanding eligibility for DST (category 3 , adjusted OR $(\mathrm{aOR})=2.57$, 95\% CI: 1.20 to 5.51) had a significantly higher likelihood of being registered compared with patients not provided with GeneXpert, while this association was not significant for providing GeneXpert testing without expanding eligibility (category 2). A positive association with receiving anti-DRTB treatment was significant only for the policy combination of providing GeneXpert and expanding eligibility for both DST and registration (category 4, aOR=2.38, 95\% CI: 1.19 to 4.79). Not surprisingly, MDR/ XDRTB patients were much more likely to be registered compared with RMR patients due to the registration policy in earlier time periods. In addition, patients with registered residence inside Zhejiang were more likely to be registered $(\mathrm{aOR}=1.96,95 \% \mathrm{CI}: 1.52$ to 2.52$)$ or treated (aOR=3.83, 95\% CI: 2.78 to 5.28). Older age was associated with lower likelihood both of being registered $(\mathrm{aOR}=0.69,95 \%$ CI: 0.53 to 0.90$)$ and of receiving antiDRTB treatment (aOR $=0.40,95 \%$ CI: 0.30 to 0.52 ).

Table 5 shows the results of the mixed-effect two-level logistic regression analysis of factors associated with the likelihood of completing treatment for patients who initiated treatment before 2017. All prefectures had some kind of financing policies in 2015 and 2016, and most prefectures had not yet expanded the eligibility of patients for DST or registration. We therefore used the individual level data on utilisation of rapid DST to assess the impact of rapid testing on treatment completion. After adjusting for other factors, older patients were less likely to complete treatment $(\mathrm{aOR}=0.24, \mathrm{p}<0.001)$, while registered residence inside Zhejiang was associated with higher probability of completing treatment $(\mathrm{aOR}=1.92$, $\mathrm{p}=0.04$ ). These two factors had significant impacts of the same direction on the registration, treatment initiation and treatment completion of RR/MDRTB patients. Utilisation of rapid DST was not associated with treatment completion in this study.

\section{DISCUSSION}

Results from this study clearly revealed the positive impacts of the combined policy changes regarding DST of presumptive DRTB patients and registration for proper management of diagnosed patients. However, inequity challenges remain in terms of servicing vulnerable groups, for example, migrant workers and the older population, in the registration, treatment and management of DRTB. 
Table 4 Factors associated with registration for diagnosed RRTB patients, and with receiving anti-DRTB treatment for registered RRTB patients

\begin{tabular}{|c|c|c|c|c|c|c|c|c|}
\hline & \multicolumn{4}{|c|}{ Registration ( $n=2367$ ) } & \multicolumn{4}{|c|}{ Receiving treatment $(n=1824)$} \\
\hline & OR & $P>z$ & \multicolumn{2}{|l|}{$95 \% \mathrm{Cl}$} & OR & $P>z$ & \multicolumn{2}{|c|}{$95 \% \mathrm{Cl}$} \\
\hline \multicolumn{9}{|l|}{ Age } \\
\hline$\geq 60$ & 0.69 & 0.006 & 0.53 & 0.90 & 0.40 & 0.000 & 0.30 & 0.52 \\
\hline \multicolumn{9}{|l|}{ Gender } \\
\hline Male & 1.08 & 0.554 & 0.85 & 1.37 & 0.87 & 0.425 & 0.62 & 1.22 \\
\hline \multicolumn{9}{|l|}{ DR type } \\
\hline MDR/XDRTB & 5.93 & 0.000 & 3.10 & 11.36 & 0.91 & 0.789 & 0.45 & 1.84 \\
\hline \multicolumn{9}{|l|}{ Patient type } \\
\hline New patient & 1.22 & 0.146 & 0.93 & 1.59 & 0.52 & 0.000 & 0.37 & 0.72 \\
\hline Treatment history & NA & & & & & & & \\
\hline No treatment & & & & & ref. & & & \\
\hline First-line drug only & & & & & 0.58 & 0.044 & 0.34 & 0.99 \\
\hline Second-line drug used & & & & & 1.11 & 0.748 & 0.58 & 2.12 \\
\hline \multicolumn{9}{|l|}{ Policy category } \\
\hline 1 & ref. & & & & ref. & & & \\
\hline 2 & 1.18 & 0.626 & 0.61 & 2.29 & 0.87 & 0.776 & 0.34 & 2.25 \\
\hline 3 & 2.57 & 0.015 & 1.20 & 5.51 & 1.44 & 0.257 & 0.77 & 2.68 \\
\hline 4 & 2.08 & 0.054 & 0.99 & 4.37 & 2.38 & 0.015 & 1.19 & 4.79 \\
\hline \multicolumn{9}{|l|}{ Registered residence } \\
\hline In Zhejiang & 1.96 & 0.000 & 1.52 & 2.52 & 3.83 & 0.000 & 2.78 & 5.28 \\
\hline \multicolumn{9}{|l|}{ Job category } \\
\hline Farmers & ref. & & & & ref. & & & \\
\hline Unemployed & 1.21 & 0.017 & 1.04 & 1.42 & 0.90 & 0.651 & 0.58 & 1.40 \\
\hline Other jobs & 0.92 & 0.281 & 0.80 & 1.07 & 0.94 & 0.784 & 0.60 & 1.47 \\
\hline \multicolumn{9}{|l|}{ Per capita GDP } \\
\hline Highest group & ref. & & & & ref. & & & \\
\hline Middle group & 1.72 & 0.122 & 0.87 & 3.42 & 2.20 & 0.025 & 1.10 & 4.38 \\
\hline Lowest group & 1.70 & 0.099 & 0.90 & 3.20 & 1.15 & 0.643 & 0.63 & 2.12 \\
\hline _cons & 0.32 & 0.002 & 0.16 & 0.66 & 1.85 & 0.207 & 0.71 & 4.78 \\
\hline prefecture var(_cons) & 0.18 & & 0.09 & 0.39 & 0.18 & & 0.03 & 0.92 \\
\hline
\end{tabular}

DRTB, drug-resistant tuberculosis; GDP, Gross Domestic Product

; MDR, multidrug resistant; RRTB, rifampicin-resistant tuberculosis; XDRTB, extensive drug resistance tuberculosis.

\section{Effectiveness and challenges of the policy interventions}

The expansion of the eligibility criteria of presumptive DRTB patients referred for DST, together with the increased funding support to equip the facilities and guarantee the supply of reagents for rapid DST like GeneXpert, has greatly improved the capacity for DRTB case finding. The significant effects of the combined policies compared with providing GeneXpert alone indicates that in order to effectively improve case finding we need to not just introduce new technologies, but also support and expand their use. The inclusion of RMR in the registration and management of DRTB patients closed the management gap between RMR and MDR/XDRTB patients, and also improved the treatment rate for $\mathrm{RR} /$ MDRTB.

It is obvious that these reforms in the DRTB policies and regulations have effectively changed the practices in DRTB control. Nevertheless, no policies aiming at migrants were issued between 2015 and 2018, and during these 4 years the degree of inequity between local and migrant patients did not seem to be mitigated either. The improved insurance benefit package was only available for those with public health insurance enrolment in Zhejiang, and in many prefectures receiving medical assistance still required local registered residence during 2015-2018. In addition, older people were less likely to 
Table 5 Factors associated with the likelihood of completing treatment for patients that initiated treatment before 2017

\begin{tabular}{|c|c|c|c|c|}
\hline & OR & $P$ value & $95 \% \mathrm{Cl}$ & Interval \\
\hline \multicolumn{5}{|l|}{ Age } \\
\hline$\geq 60$ & 0.24 & $<0.001$ & 0.14 & 0.42 \\
\hline \multicolumn{5}{|l|}{ Gender } \\
\hline Male & 0.79 & 0.442 & 0.44 & 1.44 \\
\hline \multicolumn{5}{|l|}{ Drug-resistance type } \\
\hline MDR/XDRTB & 0.50 & 0.086 & 0.23 & 1.10 \\
\hline \multicolumn{5}{|l|}{ Patient type } \\
\hline New patient & 1.06 & 0.820 & 0.64 & 1.77 \\
\hline \multicolumn{5}{|l|}{ TB treatment history } \\
\hline No treatment history & Ref. & & & \\
\hline First-line drug only & 0.74 & 0.355 & 0.39 & 1.40 \\
\hline Second-line drug used & 1.07 & 0.847 & 0.56 & 2.02 \\
\hline \multicolumn{5}{|l|}{ Test type } \\
\hline Fast test & 0.70 & 0.096 & 0.46 & 1.06 \\
\hline \multicolumn{5}{|l|}{ Registered residence } \\
\hline In Zhejiang & 1.92 & 0.040 & 1.03 & 3.59 \\
\hline \multicolumn{5}{|l|}{ Job category } \\
\hline Farmers & Ref. & & & \\
\hline Unemployed & 0.84 & 0.635 & 0.42 & 1.71 \\
\hline Other jobs & 1.44 & 0.278 & 0.74 & 2.79 \\
\hline \multicolumn{5}{|l|}{ Per capita GDP } \\
\hline Highest group & Ref. & & & \\
\hline Middle group & 0.94 & 0.939 & 0.20 & 4.39 \\
\hline Lowest group & 1.07 & 0.921 & 0.30 & 3.74 \\
\hline \multicolumn{5}{|l|}{ Year of sending sample } \\
\hline 2015 & Ref. & & & \\
\hline 2016 & 1.23 & 0.164 & 0.92 & 1.64 \\
\hline _cons & 5.58 & 0.001 & 2.01 & 15.50 \\
\hline prefecture var(_cons) & 0.21 & & 0.04 & 1.10 \\
\hline
\end{tabular}

MDR, multidrug resistant; XDRTB, extensive drug-resistance tuberculosis.

be registered or receive treatment. This may be because they were more likely to give up treatment due to the high cost and long course of the standard treatment, and health workers may not register them once they refused to provide information necessary for registration. All these findings were consistent with previous studies that age and migration for work, as well as health system factors such as lack of clear eligibility criteria for DST and limited capacity to provide DST were associated with attrition at different stages of the cascade. ${ }^{14} 1631-33$ Nevertheless, no impacts were observed of other factors, such as association between treatment experience and treatment outcome, and data on other socioeconomic factors such as financial difficulties were not available in this study.

\section{Equity challenges regarding migrant populations}

The growing number of migrants in China and other parts of the world are posing a challenge to TB control. ${ }^{31}{ }^{34-36}$
Although one systematic review found no significant differences in treatment adherence between migrant and long-term resident MDRTB patients, ${ }^{37}$ several studies identified barriers for migrants to accessing TB diagnosis and care mainly in terms of knowledge gaps and financial difficulty, ${ }^{38} 39$ and these barriers would likely to be more substantial for migrant MDRTB patients. In our study, only around $40 \%$ of the DRTB patients had a formal job other than farmers or self-employment. In China, people with a formal job will be compulsively enrolled in the urban employee basic medical insurance at the place of work as required by law. However, migrants without a formal job often choose to participate in the health insurance for residents in their hometown, which requires lower premium compared with the more developed region they migrated to, and thus cannot benefit from the reimbursement policy in their working place. Besides, they often 
have to pay the full cost of treatment first before they get reimbursed when they return home. Therefore, it is expected that many of these DRTB patients would choose to go back to their hometown for treatment, or even refuse treatment. The need for migrant DRTB patients to travel long distances and the substandard or even absent treatment received would increase the risk of disease transmission and treatment failure. A study in Shanghai, a well-developed city in eastern China, showed that financial incentives were effective for migrant TB patients to complete treatment. ${ }^{40}$ In terms of these findings, policies aimed at encouraging migrant DRTB patients to be treated and managed at their current place of residence need to be developed, and this is particularly urgent for regions with a large migrant population.

\section{Limitations}

One major limitation of our study is that almost all prefectures had some form of financing policy since 2015 and there were no individual-level data on whether the patient benefited from these financing policies. Therefore, in this study, the impacts of these financing policies could not be explored. Furthermore, other potentially important socioeconomic factors like education and income were not available in our dataset, limiting the scope of the equity analysis. Nevertheless, these limitations would not influence our conclusions that changes in the screening and registration policies of DRTB patients have largely increased the case finding and management capacity of DRTB patients, while during the period 2015-2018 differences between the local and migrant patients in the registration, treatment initiation and treatment outcome remained. We did not consider population changes either as the resident population only increased 5\% from 2015 to 2018 in Zhejiang. ${ }^{41}$ Future research should collect more individual-level data on the implementation of the health insurance and medical assistance policies to investigate the impacts of these demand-side policies on DRTB treatment more directly, and explore what kind of financing policies provided for migrants could increase their likelihood of initiating and completing anti-DRTB treatment.

\section{Author affiliations}

${ }^{1}$ Global Health Research Center, Duke Kunshan University, Kunshan, China ${ }^{2}$ Zhejiang Provincial Center for Disease Control and Prevention, Hangzhou, China ${ }^{3}$ School of Business and Economics, Vrije Universiteit Amsterdam, Amsterdam, The Netherlands

${ }^{4}$ Duke Global Health Institute, Duke University, Durham, North Carolina, USA ${ }^{5}$ National Center for Tuberculosis Control and Prevention, Centers for Disease Control and Prevention, Beijing, China

${ }^{6}$ Department of Global Health, Amsterdam Institute for Global Health and Development, Amsterdam University Medical Centres, Duivendrecht, The Netherlands

Acknowledgements This paper is part of the outputs emanating from the program entitled 'China National Health and Family Planning Commission and the Gates Foundation TB Project (Phase III)'-a collaboration between the Government of China and the Bill and Melinda Gates Foundation, and implemented by the China Center of Disease Control and Prevention (CDC). The authors of the paper also gratefully acknowledge the officers in Zhejiang Provincial CDC and the 11 prefectural CDCs for helping with collecting drug-resistant tuberculosis related policies in Zhejiang.

Contributors The study was designed by WJ, ST, CE and FC. FH, YP, XW, BC and WJ coordinated and conducted data collection. WJ conducted the literature review and wrote the manuscript as the first author. ST, CE, BC and FC provided suggestions on data analysis framework and data interpretation, and also revised the manuscript. All authors reviewed the draft manuscript, provided comments on the finalisation of the manuscript and have read and approved the manuscript in its current state.

Funding The work was supported by the Bill and Melinda Gates Foundation (grant number: OPP1149395).

Competing interests None declared.

Patient consent for publication Not required.

Ethics approval This study is under the overall evaluation study of China-Gates TB Project Phase III and used data collected from this project. The implementation of this project has received the ethical approval from China Center of Disease Control and Prevention (number: 201626). The protocol of the overall study design and data collection tools was reviewed and approved by the Institutional Review Board of Duke University (IRB approval code: 2017-0768). This paper does not use data involving human participants.

Provenance and peer review Not commissioned; externally peer reviewed.

Data availability statement Data may be obtained from a third party and are not publicly available. The data from the Tuberculosis Information Management System used in this study are owned by China CDC. The data could only be accessed after obtaining permission from China CDC.

Supplemental material This content has been supplied by the author(s). It has not been vetted by BMJ Publishing Group Limited (BMJ) and may not have been peer-reviewed. Any opinions or recommendations discussed are solely those of the author(s) and are not endorsed by BMJ. BMJ disclaims all liability and responsibility arising from any reliance placed on the content. Where the content includes any translated material, BMJ does not warrant the accuracy and reliability of the translations (including but not limited to local regulations, clinical guidelines, terminology, drug names and drug dosages), and is not responsible for any error and/or omissions arising from translation and adaptation or otherwise.

Open access This is an open access article distributed in accordance with the Creative Commons Attribution 4.0 Unported (CC BY 4.0) license, which permits others to copy, redistribute, remix, transform and build upon this work for any purpose, provided the original work is properly cited, a link to the licence is given, and indication of whether changes were made. See: https://creativecommons.org/ licenses/by/4.0/.

\section{ORCID iD}

Weixi Jiang http://orcid.org/0000-0002-5643-6255

\section{REFERENCES}

1 World Health Organization. Global tuberculosis report 2019. Geneva; 2019. 978-92-4-156571-4

2 BY L, Shi WP, Zhou CM. Rising challenge of multidrug-resistant tuberculosis in China: a predictive study using Markov modeling. Infect Dis Poverty 2020;9:1-8.

3 World Health Organization. Sustainable development goals (SDGs), 2020. Available: https://www.who.int/sdg/targets/en/

4 Fitzpatrick $\mathrm{C}$, Floyd K. A systematic review of the cost and cost effectiveness of treatment for multidrug-resistant tuberculosis. Pharmacoeconomics 2012;30:63-80.

5 Li R, Ruan Y, Sun Q, et al. Effect of a comprehensive programme to provide universal access to care for sputum-smear-positive multidrug-resistant tuberculosis in China: a before-and-after study. Lancet Glob Health 2015;3:e217-28.

6 Long Q, Qu Y, Lucas $\mathrm{H}$. Drug-Resistant tuberculosis control in China: progress and challenges. Infect Dis Poverty 2016;5:9.

7 Hutchison C, Khan MS, Yoong J, et al. Financial barriers and coping strategies: a qualitative study of accessing multidrug-resistant tuberculosis and tuberculosis care in Yunnan, China. BMC Public Health 2017;17:1-11.

8 Wingfield T, Boccia D, Tovar M, et al. Defining catastrophic costs and comparing their importance for adverse tuberculosis outcome with multi-drug resistance: a prospective cohort study, Peru. PLoS Med 2014;11:e1001675. 
9 Pan $\mathrm{H}-\mathrm{Q}$, Bele S, Feng $\mathrm{Y}$, et al. Analysis of the economic burden of diagnosis and treatment of tuberculosis patients in rural China. Int $J$ Tuberc Lung Dis 2013;17:1575-80.

10 Wang Y, McNeil EB, Huang Z, et al. Household financial burden among multidrug-resistant tuberculosis patients in Guizhou Province, China: a cross-sectional study. Medicine 2020;99:e21023.

11 Tang S, Wang L, Wang $\mathrm{H}$, et al. Access to and affordability of healthcare for TB patients in China: issues and challenges. Infect Dis Poverty 2016;5:1-5.

12 Shewade HD, Nair D, Klinton JS, et al. Low pre-diagnosis attrition but high pre-treatment attrition among patients with MDR-TB: an operational research from Chennai, India. J Epidemiol Glob Health 2017;7:227-33

13 Boyd R, Ford N, Padgen P, et al. Time to treatment for rifampicinresistant tuberculosis: systematic review and meta-analysis. Int $J$ Tuberc Lung Dis 2017;21:1173-80.

$14 \mathrm{Xu} \mathrm{C}$, Li R, Shewade HD, et al. Attrition and delays before treatment initiation among patients with MDR-TB in China (2006-13): magnitude and risk factors. PLoS One 2019;14:e0214943-16.

15 Hirasen K, Berhanu R, Evans D, et al. High rates of death and loss to follow-up by 12 months of rifampicin resistant TB treatment in South Africa. PLoS One 2018;13:e0205463-14.

16 Shewade D. High pre-diagnosis attrition among patients with presumptive MDR-TB: an operational research from Bhopal district, India. BMC Health Serv Res 2017;17:1-10.

17 Oo T, Kyaw KWY, Soe KT, et al. Magnitude and reasons for pre-diagnosis attrition among presumptive multi-drug resistant tuberculosis patients in Bago region, Myanmar: a mixed methods study. Sci Rep 2019;9:1-10.

18 Xu C, Pang Y, Li R, et al. Clinical outcome of multidrug-resistant tuberculosis patients receiving standardized second-line treatment regimen in China. $J$ Infect 2018;76:348-53.

19 Abubakar I, Zignol M, Falzon D, et al. Drug-Resistant tuberculosis: time for visionary political leadership. Lancet Infect Dis 2013;13:529-39.

20 Radin E, Ariana P, Broekel T, et al. Analyzing demand-side efficiency in global health: an application to maternal care in Vietnam. Health Policy Plan 2016;31:1281-90.

21 Boehme CC, Nicol MP, Nabeta P, et al. Feasibility, diagnostic accuracy, and effectiveness of decentralised use of the Xpert MTB/ RIF test for diagnosis of tuberculosis and multidrug resistance: a multicentre implementation study. Lancet 2011;377:1495-505.

22 Christopher PM, Widysanto A, Cucunawangsih WA, Cucunawangsih. GeneXpert Mycobacterium tuberculosis/rifampicin assay for molecular epidemiology of rifampicin-Resistant Mycobacterium tuberculosis in an Urban Setting of Banten province, Indonesia. Int J Mycobacteriol 2019;8:351-8.

23 Atashi S, Izadi B, Jalilian S, et al. Evaluation of GeneXpert MTB/RIF for determination of rifampicin resistance among new tuberculosis cases in West and northwest Iran. New Microbes New Infect 2017;19:117-20.

24 Pang Y, Xia H, Zhang Z, et al. Multicenter evaluation of GeneChip for detection of multidrug-resistant Mycobacterium tuberculosis. J Clin Microbiol 2013;51:1707-13.

25 Yin J, Yuan J, Hu Y, et al. Association between directly observed therapy and treatment outcomes in multidrug-resistant tuberculosis: a systematic review and meta-analysis. PLoS One 2016;11:e0150511-4.

26 Alipanah N, Jarlsberg L, Miller C, et al. Adherence interventions and outcomes of tuberculosis treatment: a systematic review and meta-analysis of trials and observational studies. PLoS Med 2018;15:e1002595

27 Baral SC, Aryal Y, Bhattrai R, et al. The importance of providing counselling and financial support to patients receiving treatment for multi-drug resistant TB: mixed method qualitative and pilot intervention studies. BMC Public Health 2014;14:46.

28 Wang L, Li R, Xu C, et al. The global fund in China: multidrugresistant tuberculosis nationwide programmatic scale-up and challenges to transition to full country ownership. PLoS One 2017;12:e0177536-13

29 Subbaraman R, Nathavitharana RR, Mayer KH, et al. Constructing care cascades for active tuberculosis: a strategy for program monitoring and identifying gaps in quality of care. PLoS Med 2019;16:e1002754-18.

30 stats.gov.cn. China national Bureau of statistics, 2020. Available: http://www.stats.gov.cn/tjsj/ndsj/2019/indexch.htm

31 Truzyan N, Crape B, Grigoryan R, et al. Increased risk for multidrugresistant tuberculosis in migratory workers, Armenia. Emerg Infect Dis 2015;21:474-6.

32 Parmar MM, Sachdeva KS, Dewan PK, et al. Unacceptable treatment outcomes and associated factors among India's initial cohorts of multidrug-resistant tuberculosis (MDR-TB) patients under the revised national TB control programme (2007-2011): Evidence leading to policy enhancement. PLoS One 2018;13:e0193903-24.

33 Lalor MK, Greig J, Allamuratova S, et al. Risk factors associated with default from multi- and extensively drug-resistant tuberculosis treatment, Uzbekistan: a retrospective cohort analysis. PLoS One 2013;8:e78364

34 Li X, Yang Q, Feng B, et al. Tuberculosis infection in rural labor migrants in Shenzhen, China: emerging challenge to tuberculosis control during urbanization. Sci Rep 2017;7:1-8.

35 Zumla A, Abubakar I. Improving access to multi-drug resistant tuberculosis diagnostic and health services for refugees and migrants. BMC Med 2018;16:7-10.

36 Wang W, Wang J, Zhao Q, et al. Contribution of rural-to-urban migration in the prevalence of drug resistant tuberculosis in China. Eur J Clin Microbiol Infect Dis 2011;30:581-6.

37 Nellums LB, Rustage K, Hargreaves S, et al. Multidrug-Resistant tuberculosis treatment adherence in migrants: a systematic review and meta-analysis. BMC Med 2018;16:1-11.

38 Long Q, Li Y, Wang Y, et al. Barriers to accessing TB diagnosis for rural-to-urban migrants with chronic cough in Chongqing, China: a mixed methods study. BMC Health Serv Res 2008;8:1-10.

39 Wei X, Chen J, Chen P, et al. Barriers to TB care for rural-to-urban migrant TB patients in Shanghai: a qualitative study. Trop Med Int Heal 2009;14:754-60.

40 Wei X, Zou G, Yin J, et al. Providing financial incentives to rural-tourban tuberculosis migrants in Shanghai: an intervention study. Infect Dis Poverty 2012;1:9-8.

41 Zhejiang Bureau of Statistics. Statistical Communique on the provincial economy and social development of Zhejiang in 2015 and 2018, 2021. Available: http://tjj.zj.gov.cn/col/col1229129205/index. html 